\title{
A Rotating Black Ring Solution in Five Dimensions
}

\author{
Roberto Emparan ${ }^{1, *}$ and Harvey S. Reall ${ }^{2}$ \\ ${ }^{1}$ Theory Division, CERN, CH-1211 Geneva 23, Switzerland \\ ${ }^{2}$ Physics Department, Queen Mary College, Mile End Road, London E1 4NS, United Kingdom
}

(Received 8 November 2001; published 21 February 2002)

\begin{abstract}
The vacuum Einstein equations in five dimensions are shown to admit a solution describing a stationary asymptotically flat spacetime regular on and outside an event horizon of topology $S^{1} \times S^{2}$. It describes a rotating "black ring." This is the first example of a stationary asymptotically flat vacuum solution with an event horizon of nonspherical topology. The existence of this solution implies that the uniqueness theorems valid in four dimensions do not have simple five-dimensional generalizations. It is suggested that increasing the spin of a spherical black hole beyond a critical value results in a transition to a black ring, which can have an arbitrarily large angular momentum for a given mass.
\end{abstract}

DOI: 10.1103/PhysRevLett.88.101101

Black holes in four spacetime dimensions are highly constrained objects. A number of classical theorems show that a stationary, asymptotically flat, vacuum black hole is completely characterized by its mass and spin [1], and event horizons of nonspherical topology are forbidden [2].

In this Letter we show explicitly that in five dimensions the situation cannot be so simple by exhibiting an asymptotically flat, stationary, vacuum solution with a horizon of topology $S^{1} \times S^{2}$ : a black ring. The ring rotates along the $S^{1}$ and this balances its gravitational self-attraction. The solution is characterized by its mass $M$ and spin $J$. The black hole of [3] with rotation in a single plane (and horizon of topology $S^{3}$ ) can be obtained as a branch of the same family of solutions. We show that there exist black holes and black rings with the same values of $M$ and $J$. They can be distinguished
PACS numbers: 04.50.+h, 04.20.Jb, 04.70.Bw

by their topology and by their mass dipole measured at infinity. This shows that there is no obvious fivedimensional analog of the uniqueness theorems.

$S^{1} \times S^{2}$ is one of the few possible topologies for the event horizon in five dimensions that was not ruled out by the analysis in [4] (although this argument does not apply directly to our black ring because it assumes time symmetry). An explicit solution with a regular (but degenerate) horizon of topology $S^{1} \times S^{2}$ and spacelike infinity with $S^{3}$ topology has been built recently in [5]. An uncharged static black ring solution is presented in [6], but it contains conical singularities. Our solution is the first asymptotically flat vacuum solution that is completely regular on and outside an event horizon of nonspherical topology.

Our starting point is the following metric, constructed as a Wick-rotated version of a solution in [7]:

$$
\begin{aligned}
d s^{2}= & -\frac{F(x)}{F(y)}\left(d t+\sqrt{\frac{\nu}{\xi_{1}}} \frac{\xi_{2}-y}{A} d \psi\right)^{2} \\
& +\frac{1}{A^{2}(x-y)^{2}}\left[-F(x)\left(G(y) d \psi^{2}+\frac{F(y)}{G(y)} d y^{2}\right)+F(y)^{2}\left(\frac{d x^{2}}{G(x)}+\frac{G(x)}{F(x)} d \phi^{2}\right)\right],
\end{aligned}
$$

where $\xi_{2}$ is defined below and

$$
F(\xi)=1-\xi / \xi_{1}, \quad G(\xi)=1-\xi^{2}+\nu \xi^{3} .
$$

The solution of [7] was obtained as the electric dual of the magnetically charged Kaluza-Klein C metric of [8]. Our metric can be related directly to the latter solution by analytic continuation. When $\nu=0$ we recover the static black ring solution of [6].

We assume that $0<\nu<\nu_{*} \equiv 2 /(3 \sqrt{3})$, which ensures that the roots of $G(\xi)$ are all distinct and real. They will be ordered as $\xi_{2}<\xi_{3}<\xi_{4}$. It is easy to establish that $-1<\xi_{2}<0<1<\xi_{3}<\xi_{4}<\frac{1}{\nu}$. A double root $\xi_{3}=\xi_{4}$ appears when $\nu=\nu_{*}$. Without loss of generality, we take $A>0$. Taking $A<0$ simply reverses the sense of rotation.

We take $x$ to lie in the range $\xi_{2} \leq x \leq \xi_{3}$ and require that $\xi_{1} \geq \xi_{3}$, which ensures that $g_{x x}, g_{\phi \phi} \geq 0$. In order to avoid a conical singularity at $x=\xi_{2}$ we identify $\phi$ with period

$$
\Delta \phi=\frac{4 \pi \sqrt{F\left(\xi_{2}\right)}}{G^{\prime}\left(\xi_{2}\right)}=\frac{4 \pi \sqrt{\xi_{1}-\xi_{2}}}{\nu \sqrt{\xi_{1}}\left(\xi_{3}-\xi_{2}\right)\left(\xi_{4}-\xi_{2}\right)} .
$$

A metric of Lorentzian signature is obtained by taking $y<\xi_{2}$. Examining the behavior of the constant $t$ slices of (1), one finds that $\psi$ must be identified with period $\Delta \psi=$ $\Delta \phi$ in order to avoid a conical singularity at $y=\xi_{2} \neq x$. Regularity of the full metric here can be demonstrated by converting from the polar coordinates $(y, \psi)$ to Cartesian coordinates - the $d t d \psi$ term can then be seen to vanish smoothly at the origin $y=\xi_{2}$.

There are now two cases of interest depending on the value of $\xi_{1}$. One of these will correspond to a black ring 
and the other to the black hole of [3] with only one nonvanishing angular momentum.

Case 1 is defined by $\xi_{1}>\xi_{3}$. In this case, $g_{\phi \phi}$ vanishes at $x=\xi_{3}$ and there will be a conical singularity there unless $\phi$ is identified with period

$$
\Delta \phi^{\prime}=\frac{4 \pi \sqrt{F\left(\xi_{3}\right)}}{\left|G^{\prime}\left(\xi_{3}\right)\right|}=\frac{4 \pi \sqrt{\xi_{1}-\xi_{3}}}{\nu \sqrt{\xi_{1}}\left(\xi_{3}-\xi_{2}\right)\left(\xi_{4}-\xi_{3}\right)} .
$$

We demand $\Delta \phi=\Delta \phi^{\prime}$ for consistency with (3). Since $\xi_{2}<\xi_{3}$, this is possible only if $\xi_{1}$ is fixed as a function of the other three roots (i.e., of $\nu$ ) as

$$
\xi_{1}=\frac{\xi_{4}^{2}-\xi_{2} \xi_{3}}{2 \xi_{4}-\xi_{2}-\xi_{3}} \quad \text { (black ring). }
$$

In this case it is easy to show that $\xi_{3}<\xi_{1}<\xi_{4}$, from which it follows that the factors of $F(x)$ in the metric are never zero. $x$ and $\phi$ parametrize a regular surface of topology $S^{2}$. The sections at constant $t, y$ have the topology of a ring $S^{1} \times S^{2} . x=\xi_{3}$ is an axis pointing "into" the ring (i.e., decreasing $S^{1}$ radius) and $x=\xi_{2}$ points out of the ring. This coordinate system is sketched for the static black ring in [6]; the case considered here is very similar.

Case 2 is defined by

$$
\xi_{1}=\xi_{3} \quad \text { (black hole). }
$$

In this case, $g_{\phi \phi}$ does not vanish at $x=\xi_{3}$, hence (4) need not be imposed. When (6) holds, the sections at constant $t, y$ have the topology of three-spheres $S^{3}$, with $\psi$ and $\phi$ being two independent rotation angles.

The following analysis applies to both cases 1 and 2 . Asymptotic infinity lies at $x=y=\xi_{2}$. Defining $\tilde{\phi}=$ $\frac{2 \pi \phi}{\Delta \phi}, \tilde{\psi}=\frac{2 \pi \psi}{\Delta \psi}$, and using the coordinate transformation

$$
\zeta=\frac{\sqrt{\xi_{2}-y}}{\tilde{A}(x-y)}, \quad \eta=\frac{\sqrt{x-\xi_{2}}}{\tilde{A}(x-y)},
$$

with $\tilde{A}=A \xi_{1} \sqrt{\nu\left(\xi_{3}-\xi_{2}\right)\left(\xi_{4}-\xi_{2}\right)} /\left[2\left(\xi_{1}-\xi_{2}\right)\right]$, the asymptotic metric is brought to the manifestly flat form

$$
d s^{2} \sim-d t^{2}+d \zeta^{2}+\zeta^{2} d \tilde{\psi}^{2}+d \eta^{2}+\eta^{2} d \tilde{\phi}^{2} .
$$

Note that the Killing vector fields $\mathbf{k}=\partial / \partial t, \mathbf{m}=\partial / \partial \tilde{\psi}$ are canonically normalized near infinity, and $\tilde{\psi}$ and $\tilde{\phi}$ both have period $2 \pi$.

The Arnowitt-Deser-Misner mass and angular momentum are

$$
\begin{gathered}
M=\frac{3 \pi}{2 G A^{2}} \frac{\xi_{1}-\xi_{2}}{\nu \xi_{1}^{2}\left(\xi_{3}-\xi_{2}\right)\left(\xi_{4}-\xi_{2}\right)}, \\
J=\frac{2 \pi}{G A^{3}} \frac{\left(\xi_{1}-\xi_{2}\right)^{5 / 2}}{\nu^{3 / 2} \xi_{1}^{3}\left(\xi_{3}-\xi_{2}\right)^{2}\left(\xi_{4}-\xi_{2}\right)^{2}} .
\end{gathered}
$$

The next limit to consider is $y \rightarrow-\infty$. Changing coordinates to $Y=-1 / y$ gives a metric regular in a neighborhood of $Y=0$. Hence there is a new region $Y<0$, in which the coordinate $y$ can be defined as $y=-1 / Y$ and the metric takes the same form as above. The metric in this region is regular in these coordinates for $y>\xi_{4}$. k becomes spacelike precisely at $Y=0$, so the region $y>\xi_{4}$ is referred to as the "ergoregion." The ergosurface at $Y=$ 0 has topology $S^{1} \times S^{2}$ in case 1 and $S^{3}$ in case 2 . In both cases, $\mathbf{m}$ remains spacelike throughout the ergoregion.

The above coordinates break down at $y=\xi_{4}$, so define new coordinates $\chi$ and $v$ by

$$
\begin{gathered}
d \chi=d \psi+\frac{\sqrt{-F(y)}}{G(y)} d y, \\
d v=d t+\sqrt{\frac{\nu}{\xi_{1}}}\left(y-\xi_{2}\right) \frac{\sqrt{-F(y)}}{A G(y)} d y,
\end{gathered}
$$

so $\chi$ is periodic with period $\Delta \chi=\Delta \psi$. In these new coordinates, the metric takes the form

$$
\begin{aligned}
d s^{2}= & -\frac{F(x)}{F(y)}\left(d v-\sqrt{\frac{\nu}{\xi_{1}}} \frac{y-\xi_{2}}{A} d \chi\right)^{2} \\
& +\frac{1}{A^{2}(x-y)^{2}}\left[F(x)\left(-G(y) d \chi^{2}+2 \sqrt{-F(y)} d \chi d y\right)+F(y)^{2}\left(\frac{d x^{2}}{G(x)}+\frac{G(x)}{F(x)} d \phi^{2}\right)\right] .
\end{aligned}
$$

This is regular at $y=\xi_{4}$ so the coordinate $y$ can now be continued into the region $y<\xi_{4}$. The surface $y=\xi_{4}$ is a Killing horizon of the Killing vector field

$$
\boldsymbol{\xi}=\frac{\partial}{\partial v}+\frac{A \sqrt{\xi_{1}}}{\sqrt{\nu}\left(\xi_{4}-\xi_{2}\right)} \frac{\partial}{\partial \chi}
$$

with surface gravity

$$
\kappa=\frac{A \sqrt{\nu}}{2} \frac{\xi_{1}\left(\xi_{4}-\xi_{3}\right)}{\sqrt{\xi_{4}-\xi_{1}}} .
$$

Outside this horizon, $\boldsymbol{\xi}=\mathbf{k}+\Omega_{H} \mathbf{m}$, where

$$
\Omega_{H}=\frac{2 \pi A \sqrt{\xi_{1}}}{\Delta \phi\left(\xi_{4}-\xi_{2}\right) \sqrt{\nu}}=\frac{A \sqrt{\nu}}{2} \frac{\xi_{1}\left(\xi_{3}-\xi_{2}\right)}{\sqrt{\xi_{1}-\xi_{2}}} .
$$

Note that $\boldsymbol{\xi}$ is tangent to the null geodesic generators of the horizon, and $\boldsymbol{\xi} \cdot \partial\left(\tilde{\psi}-\Omega_{H} t\right)=0$ on the horizon. It follows that the horizon is rotating with angular velocity $\Omega_{H}$ with respect to the inertial frame at infinity.

We have established that the solution possesses a rotating horizon. The area of a constant time slice through the horizon is

$$
\mathcal{A}=\frac{16 \pi^{2}}{A^{3}} \frac{\left(\xi_{4}-\xi_{1}\right)^{3 / 2}\left(\xi_{1}-\xi_{2}\right)}{\nu^{3 / 2} \xi_{1}^{3}\left(\xi_{4}-\xi_{3}\right)\left(\xi_{3}-\xi_{2}\right)\left(\xi_{4}-\xi_{2}\right)^{2}} .
$$

In case 1, when the regularity condition (5) is imposed, the topology of (a constant time slice through) this event horizon is $S^{1} \times S^{2}$ : it is a rotating black ring. In case 2 , 
when (6) is imposed, the horizon is a rotating three-sphere. The latter is actually the five-dimensional rotating black hole of [3], with one angular momentum parameter set to zero. To see this, change coordinates to

$$
\begin{aligned}
r^{2} & =\mu \frac{\left(\xi_{3}-y\right)\left(\xi_{4}-x\right)}{\left(\xi_{4}-\xi_{2}\right)(x-y)}, \\
\cos ^{2} \theta & =\frac{\left(\xi_{3}-y\right)\left(x-\xi_{2}\right)}{\left(\xi_{3}-\xi_{2}\right)(x-y)},
\end{aligned}
$$

and define

$$
\mu=\frac{4}{A^{2} \nu \xi_{3}^{2}\left(\xi_{4}-\xi_{2}\right)}, \quad a=\frac{2 \sqrt{\xi_{3}-\xi_{2}}}{A \sqrt{\nu} \xi_{3}\left(\xi_{4}-\xi_{2}\right)}
$$

(compare [9]). Then one recovers the five-dimensional Myers-Perry black hole in Boyer-Lindquist coordinates, $\mu$ and $a$ being the mass and rotation parameters defined in [3]. We emphasize that the above expressions for $M, J, \kappa$, $\Omega_{H}$, and $\mathcal{A}$ are valid for both the black ring and the black hole.

Physically, (5) is the condition that the rotation balances the gravitational self-attraction of the ring, and it fixes a relation between its mass, spin, and radius. There are two independent parameters for the solutions, $\nu$ and $A$. $A$ has dimensions of inverse length and sets the scale for the solution. $\nu$ is dimensionless and determines the shape of the $S^{1} \times S^{2}$ horizon. The $S^{1}$ can be characterized by the inner radius of curvature $R_{i}$ at $x=\xi_{3}$, and the outer radius $R_{o}$ at $x=\xi_{2}$. As $\nu \rightarrow 0$, both radii tend to the same value $R \rightarrow 3 J / M(\rightarrow \sqrt{2} / A)$. Also, $\Omega_{H} R \rightarrow 1$. Keeping $M$ fixed, the area of the $S^{2}$ tends to zero and $R$ tends to infinity, so small $\nu$ corresponds to a large thin ring. In Fig. 1 we plot physical quantities as a function of $\nu$ when $M$ is fixed. As $\nu \rightarrow \nu_{*}$, $\Omega_{H} \rightarrow(3 \pi / 8 G M)^{1 / 2}$. For $\nu$ near $\nu_{*}$ the ring is highly flattened.

If $\nu=\nu_{*}$, then the black ring and the black hole degenerate to the same solution with $\xi_{3}=\xi_{1}=\xi_{4}$. This is the $\mu=a^{2}$ limit of the five-dimensional rotating black hole, for which the horizon disappears and is replaced by a naked singularity.
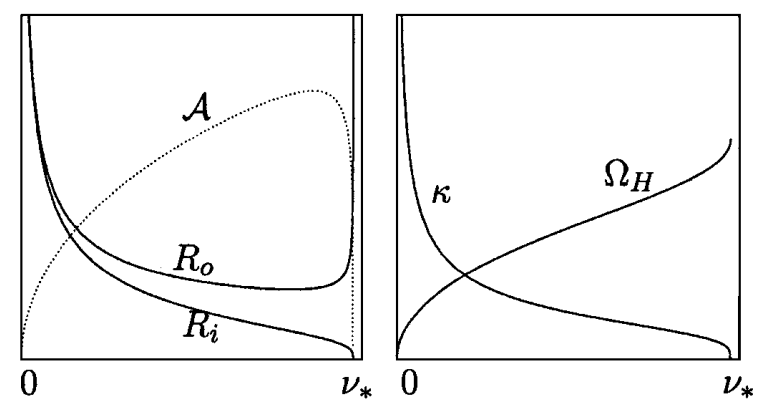

FIG. 1. Plots, as functions of $\nu$ at fixed $M$, of the inner $\left(R_{i}\right)$ and outer $\left(R_{o}\right)$ radii of curvature of the $S^{1}$, total area $\mathcal{A}$ of the ring, surface gravity $\kappa$, and angular velocity at the horizon $\Omega_{H}$. All quantities are rendered dimensionless by dividing by an appropriate power of $G M$.
The spin of the five-dimensional black holes is bounded from above [3]:

$$
\frac{J^{2}}{M^{3}} \leq \frac{32 G}{27 \pi}
$$

with equality for the (singular) $\mu=a^{2}$ solution. The corresponding ratio for the black ring solutions is

$$
\frac{J^{2}}{M^{3}}=\frac{32 G}{27 \pi} \frac{\left(\xi_{4}-\xi_{2}\right)^{3}}{\left(2 \xi_{4}-\xi_{2}-\xi_{3}\right)^{2}\left(\xi_{3}-\xi_{2}\right)} .
$$

These ratios are plotted as a function of $\nu$ in Fig. 2. Note that the angular momentum of the ring is bounded from below,

$$
\frac{J^{2}}{M^{3}}>0.8437 \frac{32 G}{27 \pi} \text {. }
$$

It is known that in six or more dimensions the spin of a black hole can be arbitrarily large [3]. In five dimensions, we have shown that the spin can also grow indefinitely, but only if the spinning object is a ring.

For $0.2164<\nu<\nu_{*}$, there are two black ring solutions with the same values of $M$ and $J$ (but different $\mathcal{A}$ ). Moreover, these satisfy the bound (20) so there is also a black hole with the same values of $M$ and $J$. This is the first explicit demonstration that the uniqueness theorems valid in four dimensions do not have a simple generalization to five dimensions.

It is straightforward to check that the classical quantities $M, J, \Omega_{H}, \kappa$, and $\mathcal{A}$ satisfy a Smarr relation

$$
M=\frac{3}{2}\left(\frac{\kappa \mathcal{A}}{8 \pi G}+\Omega_{H} J\right)
$$

Another interesting formula relates $R_{i}$ to $M$ and $\kappa$ :

$$
\kappa=\frac{3 \pi R_{i}}{8 G M} .
$$

Since the temperature of the horizon is given by $\kappa / 2 \pi$, this formula shows that the temperature of the ring is inversely proportional to its mass per unit length (around the inner $S^{1}$ ).

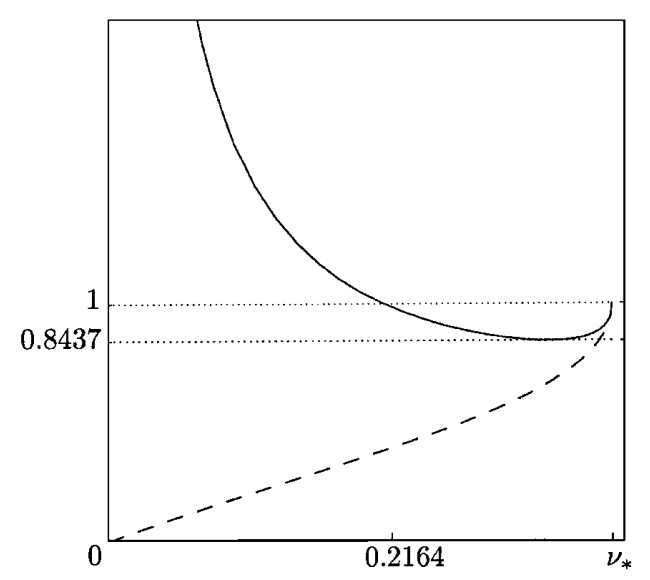

FIG. 2. $(27 \pi / 32 G) J^{2} / M^{3}$ as a function of $\nu$. Here and in the following graph, the solid line corresponds to the black ring, the dashed line to the black hole. The two dotted lines delimit the values for which a black hole and two black rings with the same mass and spin can exist. 


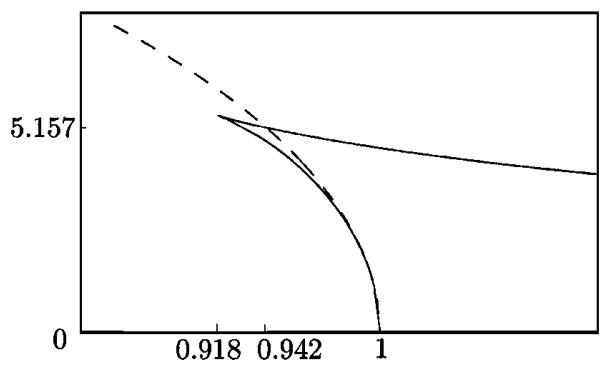

FIG. 3. $\mathcal{A} /(G M)^{3 / 2}$ against $\sqrt{27 \pi / 32 G} J / M^{3 / 2}$, around the regime in which a black hole and two black rings with the same $M$ and $J$ exist. For $\sqrt{27 \pi / 32 G} J / M^{3 / 2} \approx 0.942$ there exist a black hole and a black ring with the same mass, spin, and area $\mathcal{A} \approx 5.157(G M)^{3 / 2}$.

If one considers perturbing the black ring in such a way that it settles down to another black ring solution, then the first law of black-hole mechanics can be proved in the usual way [10] since this proof does not depend on the topology of the event horizon.

The solution with the larger area (entropy) for given values of $M$ and $J$ is the one expected to be globally thermodynamically stable in the microcanonical ensemble. In Fig. 3 we have plotted $\mathcal{A} / M^{3 / 2}$ as a function of $J / M^{3 / 2}$. As the spin increases (with the mass held fixed), there is first a small range of spins for which the black hole has larger area than both black rings. However, at a slightly larger spin, and before the singular limit is reached, the larger black ring has a greater area than the black hole and is the preferred configuration. Hence we conjecture that, as a five-dimensional black hole is spun up, a phase transition occurs from the black hole to a black ring. The singular solution is never reached.

Classically, the second law of black-hole mechanics suggests that a black hole might evolve into a black ring as angular momentum is added to it. However, this involves a change in the topology of the horizon, and it is not clear whether this is possible classically (see Ref. [11] for an example in which a classical topology change of the horizon is forbidden).

If $\nu \rightarrow 0$ at fixed $M$, then the ring becomes large and thin so one might expect ripples along the $\psi$ direction to lead to a classical Gregory-Laflamme [12] instability. However, if the above conjecture is correct, then we would expect a range of values $0<\nu_{1}<\nu<\nu_{2}<\nu_{*}$ for which the ring is classically stable. The results of [11] suggest that the horizon of unstable rings would tend to become lumpy around the $S^{1}$. The changing quadrupole moment of such an object would lead to emission of gravitational radiation until a stable end point was reached, presumably either another ring or a spherical black hole [13].
The extremal limit of the black-hole solution is a naked singularity, and it appears that the black ring plays a role in smoothing out the approach to this singularity. The existence of the ring may be related to cosmic censorship. In four dimensions, the extremal limit of the black hole is regular and the third law of black-hole mechanics forbids violation of the angular momentum bound $|J| \leq G M^{2}$ [10,14]. In dimension $D \geq 6$, black holes with a single nonvanishing angular momentum can carry arbitrarily high $J$ (for a given $M$ ). Hence cosmic censorship would not require black rings to exist in these cases. Five-dimensional black holes with $t w o$ nonzero angular momenta satisfy the bound (20) with $J$ replaced by $\left|J_{1}\right|+\left|J_{2}\right|$, but their extremal limit is nonsingular so the third law suggests that this bound cannot be violated by throwing matter into the hole. However, one might wonder what would happen if one were to throw some matter with $J_{2} \neq 0$ into a black ring (with $J_{2}=0$ ). There may be a generalization of the black ring solution that carries two angular momenta.

R. E. acknowledges partial support from UPV Grant No. 063.310-EB187/98 and CICYT AEN99-0315. H. S. R. was supported by PPARC.

*Also at Departamento de Física Teórica, Universidad del País Vasco, Bilbao, Spain.

[1] W. Israel, Phys. Rev. 164, 1776 (1967); B. Carter, Phys. Rev. Lett. 26, 331 (1971); D. C. Robinson, Phys. Rev. Lett. 34, 905 (1975).

[2] S. W. Hawking, Commun. Math. Phys. 25, 152 (1972). Details and references on topological censorship can be found in G. J. Galloway, K. Schleich, D. M. Witt, and E. Woolgar, Phys. Rev. D 60, 104039 (1999).

[3] R. C. Myers and M. J. Perry, Ann. Phys. (N.Y.) 172, 304 (1986).

[4] M.-1. Cai and G. J. Galloway, Classical Quantum Gravity 18, 2707 (2001).

[5] R. Emparan, Nucl. Phys. B610, 169 (2001).

[6] R. Emparan and H. S. Reall, hep-th/0110258.

[7] A. Chamblin and R. Emparan, Phys. Rev. D 55, 754 (1997).

[8] F. Dowker, J. P. Gauntlett, D. A. Kastor, and J. Traschen, Phys. Rev. D 49, 2909 (1994).

[9] F. Dowker, J.P. Gauntlett, G. W. Gibbons, and G. T. Horowitz, Phys. Rev. D 52, 6929 (1995).

[10] J. M. Bardeen, B. Carter, and S. W. Hawking, Commun. Math. Phys. 31, 161 (1973).

[11] G. T. Horowitz and K. Maeda, Phys. Rev. Lett. 87, 131301 (2001).

[12] R. Gregory and R. Laflamme, Phys. Rev. Lett. 70, 2837 (1993).

[13] We thank Gary Horowitz for this point.

[14] R. M. Wald, Ann. Phys. (N.Y.) 83, 548 (1974). 